\title{
Gastrointestinal stromal tumor in the rectum
}

\author{
Saika Sharmeen ${ }^{1}$, Sherry M. Zakhary ${ }^{2}$, German Torres ${ }^{1}$ \\ 1. Department of Biomedical Sciences, New York Institute of Technology, College of Osteopathic Medicine, Old Westbury, \\ New York, USA. 2. Department of Radiology, St. Barnabas Hospital, Bronx, New York, USA. \\ Correspondence: German Torres. Address: Department of Biomedical Sciences, Old Westbury, New York, 11568, USA. \\ E-mail: torresg@nyit.edu
}

Received: August 4, 2013

Accepted: September 26, 2013

Online Published: October 22, 2013

DOI : $10.5430 / j b g c . v 4 n 1 p 37$

URL: http://dx.doi.org/10.5430/jbgc.v4n1p37

\section{Abstract}

This radiology case report describes an elderly female patient diagnosed with rectal gastrointestinal stromal tumor (GIST). Although these tumors frequently occur in the gastrointestinal tract ( 70\%), they are relatively rare in the rectum ( 0.1\%). Computed tomography (CT) scans showed a tumor invading the rectal wall, radiating laterally into the posterior vaginal wall with well-defined focal necrosis around the lesions. Abdomino-perineal resection and posterior vaginectomy followed by histological and immunocytochemical analyses of biopsied tissue verified further the presence of a high grade GIST using primary antibodies directed against CD 117 and CD 34. These data support the hypothesis that GISTs are pathologically related to mutations in receptors for tyrosine kinase pathways, and suggest that elderly individuals might be at risk for developing ectopic forms of GISTs.

\section{Key words}

Cluster of differentiation, Computed tomography, Imatinib mesylate, Interstitial cells of Cajal, Receptor tyrosine kinase

\section{I ntroduction}

Gastrointestinal stromal tumors (GISTs) are common mesenchymal tumors of the gastrointestinal tract ${ }^{[1]}$. They appear to originate from the interstitial cells of Cajal in the Auerbach's nerve plexus ${ }^{[2]}$, where they often branch off to establish metastatic lesions in the stomach (70\%), small intestine (10\%-20\%) and in rare cases the esophagus and rectum ${ }^{[15,16]}$. In this regard, GISTs of the rectum account for only $0.1 \%$ of all rectal tumors ${ }^{[17]}$. The low incidence of tumorigenesis into the rectum suggests that neoplastic cells within the primary GIST favor, as a yet unidentified, microenvironment for selective growth advantage ${ }^{[13]}$. The possibility that certain microenvironments provide local selective advantages for metastatic cell growth is evidenced by the fact that even healthy cells, when seeded in suitable microenvironments, can grow into epithelial organoids with complete and functional microvasculature ${ }^{[8]}$.

GISTs are primarily driven by pathological activation of signaling transduction pathways, namely, the receptor tyrosine kinase (RTK) or the platelet-derived growth factor receptor- $\alpha$ (PDGFRA) which allow cancer cells to survive and proliferate under limited nutrient concentrations ${ }^{[20]}$. The recognition that GISTs contain activating mutations in driver genes encoding kinase receptors has led to the development of tyrosine kinase inhibitor drugs. For example imatinib mesylate (Gleevec), a proto-typical tyrosine kinase inhibitor is now mainly indicated for GIST and chronic myeloid leukemia (CML) ${ }^{[19]}$. 
Although GIST-derived metastatic lesions are primarily confined to the gastrointestinal tract, recent reports have raised the possibility that sub-clonal (i.e., heterogeneous) cells from the mutated GIST base can migrate to the esophagus, liver and anal canal ${ }^{[15,16,22]}$. Here, we support further this possibility by presenting a case of rectal GIST diagnosed by computed tomography (CT) scans, histology and immunocytochemistry of biopsied rectal tissue.

\section{Case report}

An 87-year-old Caucasian female who initially presented with significant lower gastrointestinal bleeding, was diagnosed with a $5.3 \mathrm{~cm} \times 5.2 \mathrm{~cm} \times 6.7 \mathrm{~cm}$ rectal mass suspicious for rectal carcinoma. Biopsy revealed the mass to be a primary GIST of the rectum. The patient refused neo-adjuvant therapy with imatinib mesylate, due to the fear of renal toxicity, since she only had one kidney (unilateral nephrectomy). She returned two years later with worsening obstructive symptoms characterized mainly by constipation and perianal discomfort. CT-imaging revealed the mass to have increased dramatically in size over the past two years. The mass now measured $10.6 \mathrm{~cm} \times 9.9 \mathrm{~cm} \times 12.1 \mathrm{~cm}$ which was $3 \mathrm{~cm}$ from the left anal verge (see Figure 1). Intra-operatively the tumor was seen to be invading through the rectal wall, and focally into the posterior vaginal wall (see Figure 2). The patient underwent an abdomino-perineal resection and posterior vaginectomy with a permanent end colostomy. Histology and immunocytochemistry of the resected specimen showed the mass to be composed of predominantly epithelioid cells with focal spindle, rhabdoid and myxoid features. The tumor cells exhibited polymorphism with mitotic figures up to 12/50 HPFs (data not shown). Focal necrosis was also observed. All resection margins were negative for tumor. Immunocytochemistry demonstrated the mass to be strongly positive for CD 117 and CD 34 (see Table). The histological features and the pattern of immunoreactivity were in support of the diagnosis: High grade rectal gastrointestinal stromal tumor. The post-surgical recovery was uneventful.
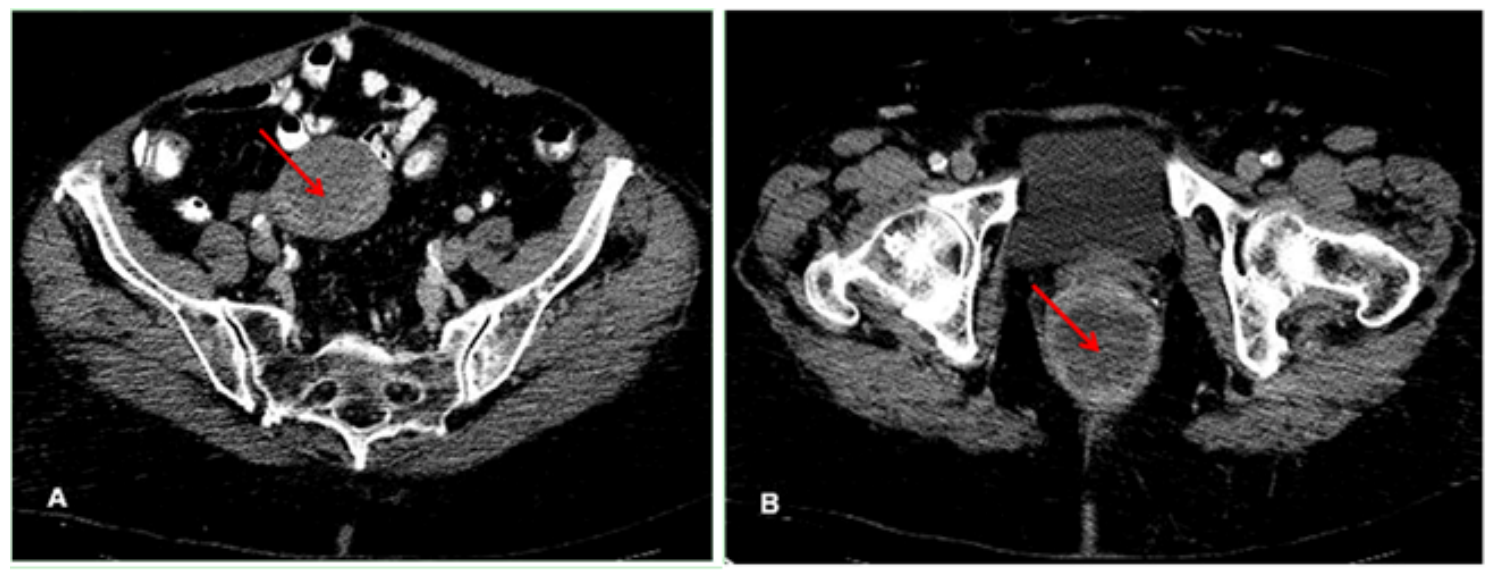

Figure 1. Contrast-enhanced CT of the abdomen/pelvis performed at the initial clinical presentation. Axial images from superior (A) and inferior (B) aspects of the mass (red arrows) demonstrate a $5.3 \mathrm{~cm} \times 5.2 \mathrm{~cm} \times 6.7 \mathrm{~cm}$ well-circumscribed heterogeneous rectal tumor with areas of central necrosis, features characteristic of GIST. Differential diagnosis includes metastatic disease, lymphoma and carcinoid tumor.

Table. Panel of primary antibodies used to diagnose and verify GIST in our patient

\begin{tabular}{ll}
\hline Antibodies & Characteristic \\
\hline CD 117 & Strongly positive \\
CD 34 & Strongly positive \\
Vimentin & Positive \\
Pancytokeratin & Negative \\
Cam 5.2 & Negative \\
LCA & Negative \\
CK5/6 & Negative \\
\hline
\end{tabular}


Table. (continued.)

\begin{tabular}{ll}
\hline Antibodies & Characteristic \\
\hline S100 & Negative \\
Desmin & Negative \\
CEA & Negative \\
CD10 & Negative \\
PAX8 & Negative \\
CD31 & Negative \\
CD163 & Negative \\
Serosal muscle actin & Negative \\
Myogenin & Negative \\
Ki-67 & $30 \%$ (proliferative index) \\
Phospho-histone & $9 / 50$ HPFs (mitotic index) \\
\hline
\end{tabular}
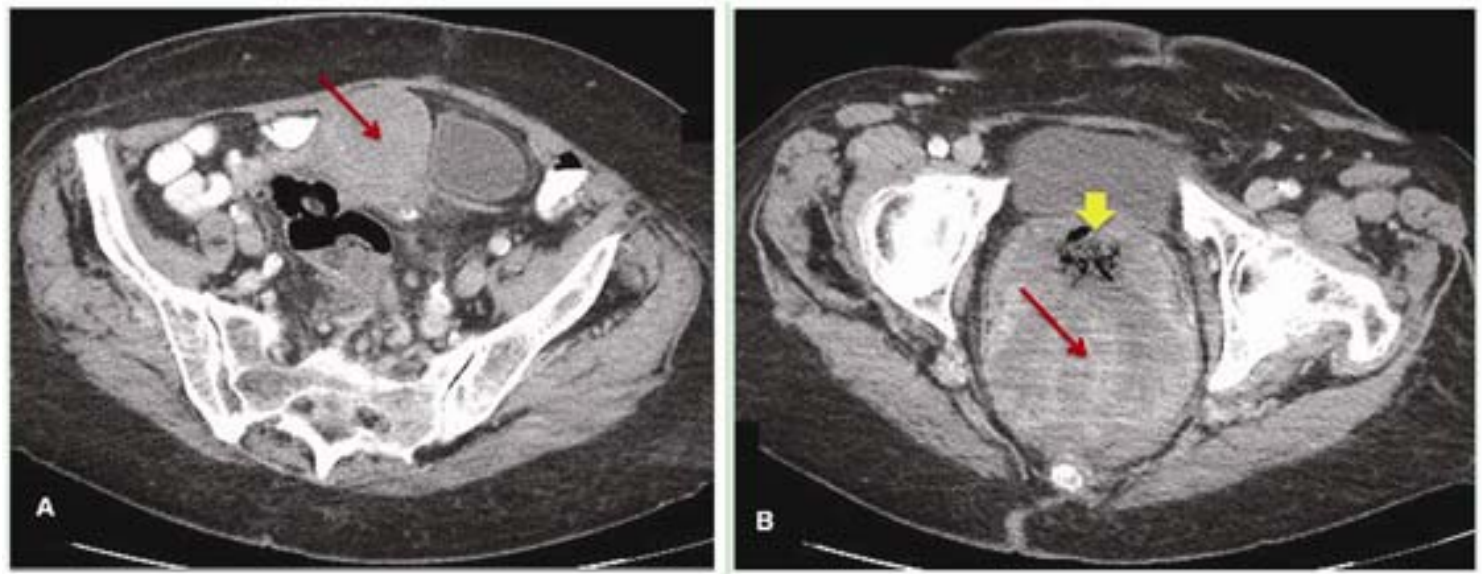

Figure 2. Contrast-enhanced CT of the abdomen/pelvis performed two years after diagnosis demonstrates interval increase in size of the rectal mass, now $10.6 \mathrm{~cm} \times 9.9 \mathrm{~cm} \times 12.1 \mathrm{~cm}$. Axial images show no significant changes in heterogeneity or central necrotic features of the rectal mass (red arrows). A: Superior aspect, B: Inferior aspect.

However the large tumor can now be seen partially occluding the rectal lumen (yellow arrow, B).

\section{Discussion}

GISTs are mesenchymal neoplasms in which mutations of driver genes (e.g., rtk) confer a selective growth advantage in thriving microenvironments of the gastrointestinal tract. Most of these mutations (> 90\%) are caused by gain-of-function mutations that result in a new function ability for the encoded protein ${ }^{[9]}$. In this particular case, the rtk gene encodes a trans-membrane receptor for growth factors that stimulate cell division, either directly or indirectly. As a result of this ability, mutated cells outgrow epithelial cells that surround gastrointestinal tissues and become microscopic clones. This process of mutation followed by clonal formation and eventually sub-clonal expansion generates a malignant tumor that can metastasize to other self-renewing organs ${ }^{[18,20]}$. The fact that genetically diverse sub-clones are found within a given tumor mass suggests that tumor heterogeneity, whether from gene mutations or abnormal levels of proteins, can diminish the metastatic lesion susceptibility to anti-cancer drugs. In other words, heterogeneity among the cells of a tumor provides the seeds for drug resistance ${ }^{[20]}$.

Against this background, our patient was diagnosed with GIST of the rectum which is a relatively rare form of stromal cancer as previously mentioned in the introduction. It should be noted that diagnosis was only based on CT scan imaging and histo-pathological examinations. Unfortunately, cancer-genome sequencing was not performed on our patient to determine how germ-line variants and mutations are associated with rectal carcinoma. The use of cancer-genome sequencing would have aided in suggesting targeted therapies for our patient (e.g., phosphatidylinositol 3-kinase 
(PI3K/Akt/mammalian target of rapamycin [mTOR] inhibitors), and would have also provided information on our patient's genetic susceptibility to GIST. Nevertheless, once GIST was diagnosed, our patient was prescribed oral formulation of imatinib mesylate (100 mg/daily). However, our patient refused therapeutic intervention due to the risk of intolerable side-effects of the drug. Imatinib mesylate is a tyrosine kinase inhibitor that targets the BCR-ABL protein in CML, $c$-kit (KIT) and PDGFRA ${ }^{[11]}$. This inhibition occurs because imatinib mesylate interferes with the catalytic activity of tyrosine kinases, for example, in phosphorylation cascades ${ }^{[20]}$. However, imatinib mesylate is notoriously non-selective and it is also prescribed by dermatologists for dermatofibrosarcoma protuberans, systemic sclerosis and systemic mastocytosis ${ }^{[12]}$. Thus, adverse skin side-effects such as edema, maculopapular rashes and Stevens-Johnson syndrome are commonly cited following imatinib mesylate therapy ${ }^{[12]}$. Our patient, however, refused drug treatment due to the fact that she had undergone unilateral nephrectomy. This appears to be a justifiable reason for non-adherence to drug therapy as several clinical cases report acute renal failure in patients taking tyrosine kinase inhibitors for CML ${ }^{[5,6,21]}$.

It is safe to argue that the risk:benefit assessment ratio from imatinib mesylate therapy could have been responsible for the significant increase in tumor mass size in our patient (from $5.3 \mathrm{~cm} \times 5.2 \mathrm{~cm} \times 6.7 \mathrm{~cm}$ to $10.6 \mathrm{~cm} \times 9.9 \mathrm{~cm} \times 12.1 \mathrm{~cm}$ in 2 years-time). Indeed, current imatinib msylate therapy often shows adequate results in the management and outcome of GISTs ${ }^{[14]}$. But another possible explanation is that as our patient was advanced in age (87-years-old), her solid, heterogeneous tumor most likely contained twice as many mutations as a morphologically identical solid tumor in a younger patient (e.g., a 50-year-old woman). Thus, age of the patient, developmental stage of the tumor and epigenetic changes (i.e., methylation) of the carcinoma, could have contributed to the increased neoplastic state of GIST, rather than non-adherence to oral anti-cancer therapy in general.

As most solid tumors of size visible on CT scan imaging are thought to be resistant to virtually any prophylactic drug agent ${ }^{[3,4,7]}$, our patient underwent an abdomino-perineal resection and posterior vaginectomy of rectal GIST. Though the primary tumor was removed, it is understood that metastatic lesions with heterogeneous sub-clonal cells cannot be removed by surgery and must be treated with oral therapies. However as indicated earlier, our patient refused imatinib mesylate therapy as GISTs patients under this drug regimen are at risk of developing tubulointerstitial nephropathy ${ }^{\text {[5, } 6,21]}$. This severe adverse drug effect is most likely caused by differential inhibition of related tyrosine kinases in the kidney. If renal failure is reported in such cancer patients, kidney dialysis should be considered as a disease-management strategy.

\section{Conclusion}

In conclusion, we have described here a clinical presentation of GIST in the rectum. Although relatively rare, GIST formations distal from the alimentary canal are being reported more frequently. A major unresolved issue swirling around this clinical presentation is whether rectal GIST is an ectopic occurrence directly correlated with age, or whether it is a cancer that metastasizes from interstitial cells of Cajal to other self-renewing sites. In this regard, interstitial cells of Cajal or interstitial Cajal-like cells are not exclusively confined to the gut, but are also present in visceral organs that generate spontaneous rhythmic muscle contractions such as the genital system ${ }^{[10]}$. Thus, it is conceivable that GISTs may also originate within the rectum and anal canal as the aforementioned interstitial Cajal-like cells could carry oncogenes whose protein products would become abnormally active over-time. It should be noted that no two cancers have identical clinical courses, regardless of site of origin. What is known with a certain degree of confidence is that signal transduction pathways involving receptor tyrosine kinases are affected by mutations in human cancers, and that all clinically approved drugs for common forms of cancers are directed against kinases ${ }^{[20]}$. Rectal GIST should be considered in the differential diagnosis of rectal masses in elderly patients.

\section{Abbreviations}

CD (cluster of differentiation); CD 117 (or c-Kit; Gene ID: 3815) is classified as a cytokine receptor (type III) localized to the surface of hematopoietic stem cells (National Center for Biotechnology Information). This receptor subtype has a high 
affinity for stem cell factor ligands. CD 34 (Gene ID: 947) is a cluster of differentiation molecule localized to the cytoplasm which functions as a cell-cell adhesion factor (National Center for Biotechnology Information).

The cytoplasmic domain for this molecule has an intracellular consensus site for protein kinase C phosphorylation.

\section{References}

[1] Rajendra R, Pollack SM, Jones RL. Management of gastrointestinal stromal tumors. Future Oncol. 2013 Feb; 9(2): $193-206$. http://dx.doi.org/10.2217/fon.12.178

[2] Terada T. Smooth muscles and stem cells of embryonic guts express KIT, PDGFRRA, CD34 and many other stem cell antigens: suggestion that GIST arise from smooth muscles and gut stem cells. Int J Clin Exp Pathol. 2013 May 15; 6(6): $1038-45$.

[3] Diaz LA Jr, Williams RT, Wu J, Kinde I, Hecht JR, Berlin J, et al. The molecular evolution of acquired resistance to targeted EGFR blockade in colorectal cancers. Nature. 2012 Jun 28; 486(7404): 537-40.

[4] Durrett R, Moseley S. Evolution of resistance and progression to disease during clonal expansion of cancer. Theor Popul Biol. 2010 Feb; 77(1): 42-8.

[5] Gafter-Gvili A, Ram R, Gafter U, Shpilberg O, Raanani P. Renal failure associated with tyrosine kinase inhibitors-case report and review of the literature. Leuk Res. 2010 Jan; 34(1): 123-7.

[6] Holstein SA, Stokes JB, Hohl RJ. Renal failure and recovery associated with second-generation Bcr-Abl kinase inhibitors in imatinib-resistant chronic myelogenous leukemia. Leuk Res. 2009 Feb; 33(2): 344-7.

[7] Komarova NL, Wodarz D. Drug resistance in cancer: principles of emergence and prevention. Proc Natl Acad Sci U S A. 2005 Jul 5; 102(27): 9714-9.

[8] Komori J, Boone L, DeWard A, Hoppo T, Lagasse E. The mouse lymph node as an ectopic transplantation site for multiple tissues. Nat Biotechnol. 2012 Oct; 30(10): 976-83. Epub 2012 Sep 23. http://dx.doi.org/10.1038/nbt.2379

[9] Lasota J, Xi L, Coates T, Dennis R, Evbuomwan MO, Wang ZF, et al. No KRAS mutations found in gastrointestinal stromal tumors (GISTs): molecular genetic study of 514 cases. Mod Pathol. 2013 May 24. [Epub ahead of print] http://dx.doi.org/10.1038/modpathol.2013.89

[10] Min KW, Leabu M. Interstitial cells of Cajal (ICC) and gastrointestinal stromal tumor (GIST): facts, speculations, and myths. J Cell Mol Med. 2006 Oct-Dec; 10(4): 995-1013.

[11] Patel S. Exploring Novel Therapeutic Targets in GIST: Focus on the PI3K/Akt/mTOR Pathway. Curr Oncol Rep. 2013 Apr 20. [Epub ahead of print].

[12] Pretel-Irazabal M, Tuneu-Valls A, Ormaechea-Pérez N. Adverse Skin Effects of Imatinib, a Tyrosine Kinase Inhibitor Actas Dermosifiliogr. 2013 Apr 30. pii: S0001-7310(13)00108-7. [Epub ahead of print].

[13] Quante M, Varga J, Wang TC, Greten FR. The Gastrointestinal Tumor Microenvironment. Gastroenterology. 2013 Apr 10; pii: S0016-5085(13)00501-5.

[14] Al-Barrak J, Cheung WY. Adherence to imatinib therapy in gastrointestinal stromal tumors and chronic myeloid leukemia. Support Care Cancer. 2013 May 25. [Epub ahead of print]

[15] Singhal S, Singhal A, Tugnait R, Varghese V, Tiwari B, Arora PK, et al. Anorectal gastrointestinal stromal tumor: a case report and literature review. Case Rep Gastrointest Med. 2013; 2013: 934875.

[16] Sjogren PP, Banerji N, Batts KP, Graczyk MJ, Dunn DH. Rare presentation of a gastrointestinal stromal tumor with spontaneous esophageal perforation: A case report. Int J Surg Case Rep. 2013 Apr 28. pii: S2210-2612(13)00134-X.

[17] Suzuki T, Sadahiro S, Tanaka A, Okada K, Okamura H, Machida T, et al. Gastrointestinal stromal tumor of the rectum: report of three cases. Tokai J Exp Clin Med. 2011; 36(3): 58-62.

[18] Takahashi H, Shigeta T, Umeda M, Komori T. A new in vitro invasion model for oral cancer using an acellular allogenic dermal matrix (Alloderm): the relationship among in vitro invasion activity, in vivo invasion and metastasis. Kobe J Med Sci. 2012 Mar 8; 57(4): E128-36.

[19] Croom KF, Imatinib mesylate: in the treatment of gastrointestinal stromal tumours. Perry CM. Drugs. 2003; 63(5): 513-22; discussion 523-4.

[20] Vogelstein B, Papadopoulos N, Velculescu VE, Zhou S, Diaz LA Jr, Kinzler KW. Cancer genome landscapes. Science. 2013 Mar 29; 339(6127): 1546-58.

[21] Yuzawa Y, Sato W, Masuda T, Hamada Y, Tatematsu M, Yasuda Y, et al. Acute kidney injury presenting a feature of leukemic infiltration during therapy for chronic myelogenous leukemia. Intern Med. 2010; 49(12): 1139-42. PMid: 20558931. http://dx.doi.org/10.2169/internalmedicine.48.2747

[22] Zhou B, Zhang M, Yan S, Zheng S. Primary gastrointestinal stromal tumor of the liver: report of a case. Surg Today. 2013 May 17. [Epub ahead of print]. 